\title{
Teor de Sílica em Compósitos Confeccionados com Bagaço de Cana-de-açúcar e Eucalipto
}

\author{
Ugo Leandro Belini ${ }^{1}$, Mario Tomazello Filho ${ }^{1}$, Lourival Marin Mendes ${ }^{2}$, \\ Marta Karina Leite ${ }^{1}$, Paulo Milton Rodrigues de Lima ${ }^{3}$ \\ ${ }^{1}$ Departamento de Ciências Florestais, Escola Superior de Agricultura "Luiz de Queiroz" - ESALQ, \\ Universidade de São Paulo - USP \\ ${ }^{2}$ Departamento de Ciências Florestais, Universidade Federal de Lavras - UFLA \\ ${ }^{3}$ Duratex S.A.
}

\begin{abstract}
RESUMO
A inserção de insumos alternativos na confecção de novos compósitos implica em detalhada caracterização, pois podem ocorrer características negativas no seu processamento ou uso final, como a presença de sílica, ou impurezas minerais. Neste trabalho, foi determinado o teor de sílica em painéis confeccionados com diferentes percentuais de fibras de eucalipto e partículas de bagaço de cana-de-açúcar conforme metodologia vigente e comparado com a literatura especializada. Como resultado principal, o aumento do percentual de utilização do bagaço de cana-de-açúcar na matriz fibrosa de eucalipto dos compósitos promove um expressivo acréscimo do teor de sílica.
\end{abstract}

Palavras-chave: biomassa, impurezas minerais, novos produtos.

\section{Sand Content in Composites Made with Sugarcane Bagasse and Eucalypt}

\begin{abstract}
The insertion of alternative raw materials in a manufacture of new composites implies in their detailed characterization, once there may be negative characteristics to their processing or final utilization, as it is the case of the sand content or mineral impurities. In this research, the sand content of panels manufacuture with different percentages of eucalypt fibers and sugarcane bagasse particles were determined in agreement with the available methodology and then compared to those found in the specialized literature. Main results showed that the increase of sugarcane bagasse percentage in the fibrous matrix of eucalypt in composites promotes expressive increase in the sand content.
\end{abstract}

Keywords: biomass, mineral impurities, new products. 


\section{INTRODUÇÃO}

Desde o ano 2000, a produção de cana-deaçúcar vem crescendo no Brasil, a uma taxa anual de aproximadamente 35 milhões de $\mathrm{t}$ (CTC, 2011) e, de acordo com a União da Indústria de Canade-açúcar (UNICA, 2010), na safra 2008-2009, houve uma produção de 570 milhões de $t$ de canade-açúcar que resultaram em 160 milhões de t de bagaço, concentradas na região Centro-Sul. Coincidentemente, esta região possui quase a totalidade das indústrias de painéis reconstituídos de fibras e partículas do Brasil (ABIPA, 2010).

Já as florestas plantadas de eucalipto ocupam uma área de 4,5 milhões de ha (ABRAF, 2010) e estão presentes na maioria dos estados brasileiros, com destaque também, para os da região Centro-Sul. Estas florestas apresentaram crescimento de 41,1\% no período entre 2004 e 2009, resultado de um conjunto de fatores que vêm favorecendo o plantio em larga escala deste gênero. Apresentam, também, grande competitividade internacional, em qualidade e quantidade (FAO, 2000).

Pesquisas procuram utilizar resíduos industriais em processos produtivos convencionais de painéis de fibra (Mantanis et al., 2004; Athanassiadou et al., 2005). Verifica-se, no caso específico do bagacilho de cana-de-açúcar, que empresas têm apresentado projetos para a produção de painéis AFB (Agricultural Fiber Board) integrados às usinas canavieiras para o aproveitamento e agregação de valor ao bagaço excedente em Usinas de Açúcar e Álcool (Ecowood do Brasil, 2009) e que resultados de pesquisas nacionais e internacionais indicam a viabilidade de utilização do bagaço de cana-de-açúcar em diferentes matrizes poliméricas para a confecção de compósitos (Rowel \& Keany, 1991; Paiva et al., 1998; Widyorini et al., 2005; Campos et al., 2009).

Considerando-se a classificação dos componentes químicos entre o bagaço e a madeira de eucalipto, a grande diferença se encontra no teor de cinzas ou impurezas minerais. Enquanto no bagaço há grande variação de 0,7\% (Rowel \& Keany, 1991) a 5,0\% (CTC, 2011 apud Paturau, 1989), para 10 diferentes espécies de eucalipto, o teor de cinzas apresentou variação entre 0,2 a 1,0\% (Brito \& Barrichelo, 1977), percentual de variação também observado por
Forest Products Laboratory (1999) para a substância madeira.

Neste contexto, o presente trabalho visou a determinação do teor de sílica em compósitos confeccionados com diferentes percentuais das as matérias-primas (partículas de bagaço de cana-deaçúcar e fibras de eucalipto.

\section{MATERIAL E MÉTODOS}

\subsection{Obtenção do bagaço de cana-de-açúcar e das fibras de eucalipto}

As amostras de bagaço de cana-de-açúcar foram coletadas no pátio de armazenamento da Usina Açucareira São Manoel S.A., localizada no município de São Manuel, Estado de São Paulo. As amostras "in natura" foram coletadas e acondicionadas em sacos plásticos, mantidas em caixas de isopor e transportadas para o Laboratório de Anatomia e Identificação de Madeiras do Departamento de Ciências Florestais da ESALQ/ USP em Piracicaba. Nesta etapa, atentou-se para a coleta de amostras imediatamente à saída do sistema de esmagamento e obtenção do caldo, visto que as partículas de cana-de-açúcar que ficam estocadas, por exemplo, da safra do ano anterior, podem sofrer degradação. Posteriormente, o material foi seco em estufa a $105^{\circ} \mathrm{C}$ até atingir aproximadamente $5 \%$ de umidade, evitando-se o desenvolvimento de microorganismos. Para os procedimentos de encolagem e prensagem dos painéis, todo o material sofreu classificação morfológica em peneiras vibratórias, e as partículas que passavam na peneira com abertura de 2,0 $\mathrm{mm}$ foram separadas para a confecção dos painéis, etapa realizada na empresa Duratex S.A.

Os componentes fibrosos da madeira de Eucalyptus grandis foram coletados na linha industrial da empresa Duratex S.A. na cidade de Botucatu, Estado de São Paulo, após a fase de desfibramento dos cavacos de madeira e antes da etapa de encolagem.

\subsection{Confecção dos painéis}

Os painéis foram confeccionados conforme os tratamentos indicados na Tabela 1. Etapa A (inicial): 
Tabela 1. Tratamentos experimentais (Fases A e B) propostos para a confecção de painéis com fibras de eucalipto e partículas de bagaço de cana-de-açúcar.

Table 1. Experimental treatments (Phases A and B) proposed for confection panels with eucalyptus fibers and sugarcane bagasse particles.

\begin{tabular}{|c|c|c|c|c|c|c|c|}
\hline \multirow[t]{2}{*}{ Etapa } & \multirow{2}{*}{$\begin{array}{c}\text { Tratamento } \\
\left(\mathbf{N}^{\circ}\right)\end{array}$} & \multicolumn{2}{|c|}{$\begin{array}{l}\text { Fibras de } \\
\text { eucalipto }\end{array}$} & \multicolumn{2}{|c|}{$\begin{array}{c}\text { Partículas de } \\
\text { bagaço de cana }\end{array}$} & \multirow{2}{*}{$\begin{array}{c}\text { Resina } \\
\text { UF } \\
(\%)\end{array}$} & \multirow{2}{*}{$\begin{array}{c}\begin{array}{c}\text { Emulsão de } \\
\text { parafina }\end{array} \\
(\%)\end{array}$} \\
\hline & & $(\%)$ & (kg seco) & $(\%)$ & (kg seco) & & \\
\hline \multirow{5}{*}{ A } & 1 & 100 & 3,70 & 0 & 0 & \multirow{5}{*}{14} & \multirow{5}{*}{0,8} \\
\hline & 2 & 75 & 2,78 & 25 & 0,93 & & \\
\hline & 3 & 50 & 1,85 & 50 & 1,85 & & \\
\hline & 4 & 25 & 0,93 & 75 & 2,78 & & \\
\hline & 5 & 0 & 0 & 100 & 3,70 & & \\
\hline \multirow{12}{*}{ B } & 1 & 100 & 3,70 & 0 & 0 & 13 & \multirow{12}{*}{0,8} \\
\hline & 2 & 100 & 3,70 & 0 & 0 & 16 & \\
\hline & 3 & 95 & 3,52 & 5 & 0,19 & 13 & \\
\hline & 4 & 95 & 3,52 & 5 & 0,19 & 16 & \\
\hline & 5 & 90 & 3,33 & 10 & 0,37 & 13 & \\
\hline & 6 & 90 & 3,33 & 10 & 0,37 & 16 & \\
\hline & 7 & 85 & 3,15 & 15 & 0,56 & 13 & \\
\hline & 8 & 85 & 3,15 & 15 & 0,56 & 16 & \\
\hline & 9 & 80 & 2,96 & 20 & 0,74 & 13 & \\
\hline & 10 & 80 & 2,96 & 20 & 0,74 & 16 & \\
\hline & 11 & 75 & 2,78 & 25 & 0,93 & 13 & \\
\hline & 12 & 75 & 2,78 & 25 & 0,93 & 16 & \\
\hline
\end{tabular}

avaliaram-se 5 tratamentos referentes às diferentes porcentagens de mistura de partículas de bagaço de cana-de-açúcar e de fibras de eucalipto, sendo confeccionados dois painéis por tratamento, em um total de 10 painéis. Etapa B (final): avaliaram-se 12 tratamentos, referentes às diferentes porcentagens de mistura de partículas de bagaço de cana-de-açúcar e de fibras de eucalipto e diferentes porcentagens de dosagem de resina ureia-formaldeído (UF), sendo confeccionados 4 painéis por tratamento, ou seja, um total de 48 painéis.

\subsection{Determinação do teor de sílica dos painéis}

Os ensaios para determinação dos teores de sílica dos painéis foram realizados conforme norma ABNT (2006) NBR 15316-3/2006 (Painéis de Fibra de Média Densidade: Parte 3 - Métodos de Ensaio). Dos corpos de prova que foram utilizados para as avaliações mecânicas de módulo de ruptura (MOR) e módulo de elasticidade (MOE), em cada tratamento, foram obtidos $200 \mathrm{~g}$ de painéis com dimensões de 15 × 25 × $25 \mathrm{~mm}$ para determinação do teor de sílica (impurezas minerais). A presença do mineral foi identificada por imagens de microscopia eletrônica de varredura (MEV) e caracterizada quimicamente por análise elementar em EDS (Energy Dispersive Spectroscopy).

\section{RESULTADOS E DISCUSSÃO}

A Figura 1 ilustra: a) imagem MEV do dióxido de silício, ou sílica $\left(\mathrm{S}_{\mathrm{i}} \mathrm{O}_{2}\right)$, verificada em amostra da mistura de componentes fibrosos do eucalipto e do bagaço de cana-de-açúcar; e b) gráfico da análise química elementar da mesma região da imagem, indicando alto teor de sílica representado em pico do elemento $\mathrm{S}_{\mathrm{i}}$.

O aumento do teor de sílica dos painéis associado ao aumento do percentual de fibras de cana-deaçúcar é evidente na Tabela 2: painéis com 100\% de fibras de cana-de-açúcar apresentaram 3\% de sílica, enquanto que nos painéis com $100 \%$ de fibras de eucalipto esse valor diminuiu para 0,007 a 0,018\%. A literatura indica grande dispersão de valores de sílica para o bagaço de cana-de-açúcar: 0,7\% (Rowel \& Keany, 1991), 4,0\% (Chen \& Hsu, 2002), 2,5\% 

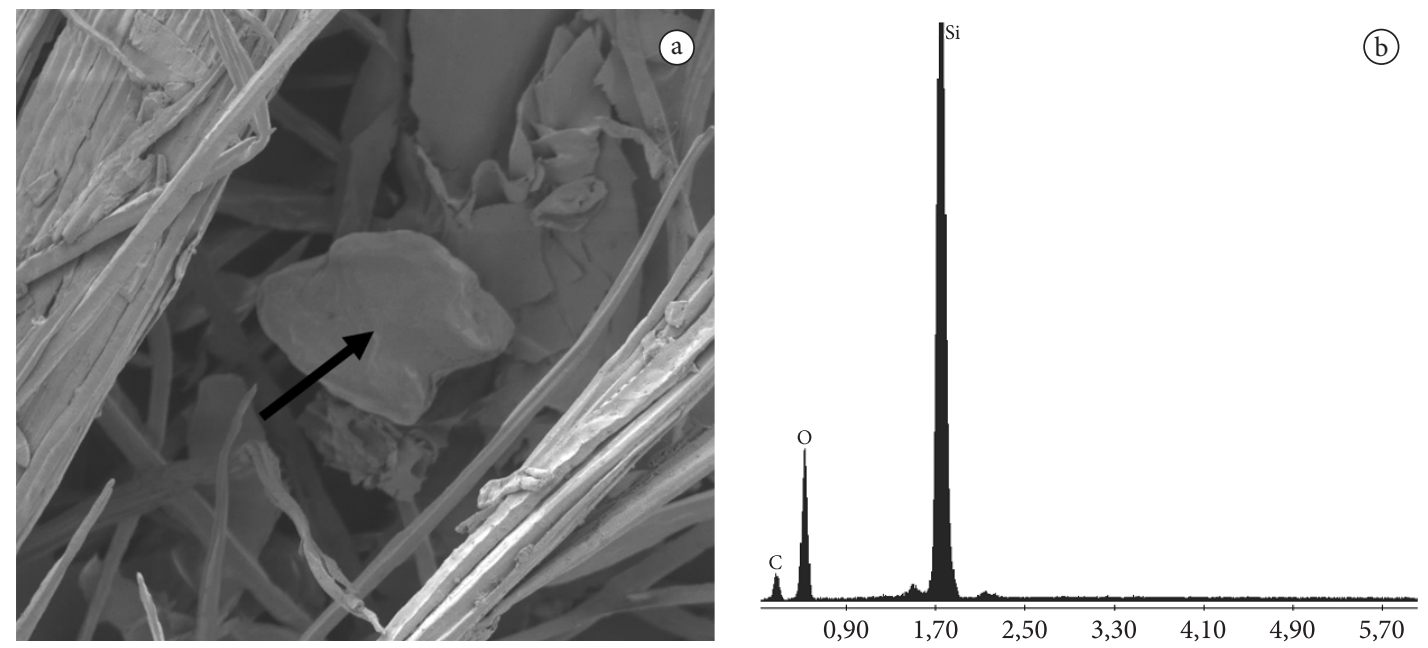

Figura 1. a) Partícula de $\mathrm{S}_{\mathrm{i}} \mathrm{O}_{2}$ identificada (seta) em imagem MEV (500x); e b) análise química elementar indicativa de pico de concentração de $\mathrm{S}_{\text {i }}$.

Figure 1. a) identified particle of $\mathrm{SiO}_{2}$ (arrow) in SEM image (500x) and b) elemental chemical analyzes indicating peak concentration of $S_{i}$.

Tabela 2. Teor de sílica (\%) de painéis confeccionados com diferentes percentuais de fibras de eucalipto e partículas de bagaço de cana-de-açúcar.

Table 2. Sand content (\%) in panels made with different percentages of eucalyptus fibers and sugarcane bagasse particles.

\begin{tabular}{|c|c|c|c|c|c|}
\hline \multirow[t]{2}{*}{ Etapa } & \multirow[t]{2}{*}{ Tratamento } & $\begin{array}{l}\text { Fibras de } \\
\text { eucalipto }\end{array}$ & $\begin{array}{c}\text { Partículas de } \\
\text { bagaço de cana }\end{array}$ & $\begin{array}{c}\text { Resina } \\
\text { UF }\end{array}$ & $\begin{array}{c}\text { Teor de } \\
\text { sílica }\end{array}$ \\
\hline & & $(\%)$ & (\%) & $(\%)$ & $(\%)$ \\
\hline \multirow{5}{*}{ A } & 1 & 100 & 0 & \multirow{5}{*}{14} & 0,007 \\
\hline & 2 & 75 & 25 & & 0,844 \\
\hline & 3 & 50 & 50 & & 1,633 \\
\hline & 4 & 25 & 75 & & 2,688 \\
\hline & 5 & 0 & 100 & & 3,049 \\
\hline \multirow{12}{*}{ B } & 1 & \multirow{2}{*}{100} & \multirow{2}{*}{0} & 13 & 0,015 \\
\hline & 2 & & & 16 & 0,018 \\
\hline & 3 & \multirow{2}{*}{95} & \multirow{2}{*}{5} & 13 & 0,206 \\
\hline & 4 & & & 16 & 0,162 \\
\hline & 5 & \multirow{2}{*}{90} & \multirow{2}{*}{10} & 13 & 0,382 \\
\hline & 6 & & & 16 & 0,273 \\
\hline & 7 & \multirow{2}{*}{85} & \multirow{2}{*}{15} & 13 & 0,606 \\
\hline & 8 & & & 16 & 0,585 \\
\hline & 9 & \multirow{2}{*}{80} & \multirow{2}{*}{20} & 13 & 0,803 \\
\hline & 10 & & & 16 & 0,764 \\
\hline & 11 & \multirow{2}{*}{75} & \multirow{2}{*}{25} & 13 & 0,850 \\
\hline & 12 & & & 16 & 0,900 \\
\hline
\end{tabular}

(Romero, 2007) e variações entre 1,5 e 4,0\% (CTC, 2011).

Em meados de 2003, quando o mercado brasileiro passou a receber painéis MDF com $100 \%$ de fibras de eucalipto, muitos usuários o consideraram mais abrasivo no processamento mecânico em equipamentos de corte, em relação aos tradicionais painéis MDF de Pinus sp. Como as espécies apresentavam teor de sílica semelhante no seu lenho, entendeu-se que a diferença era devida a sua estrutura anatômica. Porém, considerando a adição de fibras de bagaço de cana-de-açúcar, há 
um expressivo aumento do teor de sílica do painel que pode maximizar este problema. Neste contexto, sugere-se a inclusão da análise do desgaste dos materiais de corte na avaliação dos painéis de fibras e/ou de partículas de cana-de-açúcar.

Como procedimento complementar, a lavagem prévia do bagaço de cana-de-açúcar poderia ser efetiva para a retirada de impurezas minerais oriundas da colheita dos colmos, etapa esta que é comumente empregada para os cavacos de madeira de eucaliptos na confecção de painéis MDF.

Similarmente ao verificado no sistema de lavagem de cavacos de madeira para o processo de confecção de painéis MDF, o processo de lavagem de cana na mesa de alimentação para extração do caldo reduz significativamente as impurezas minerais que entram com o vegetal, principalmente da terra oriunda da colheita dos colmos.

Embora a cana inteira apresente maior quantidade de impurezas minerais do que a cana picada, a ordem se inverte quanto às cinzas no bagaço. No bagaço de cana picada, o teor de cinzas é superior àquele do bagaço de cana inteira, atingindose até $14 \%$. Isto ocorre porque normalmente a cana picada não passa pelo processo de lavagem para redução da terra aderida aos colmos, como ocorre com a cana inteira (CTC, 2011).

\section{CONCLUSÕES}

Os resultados verificados no presente trabalho permitem tecer as seguintes conclusões:

- A técnica de microscopia eletrônica de varredura (MEV) permitiu identificar elementos de sílica no interior de compósitos confeccionados com diferentes percentuais de partículas de bagaço de cana-de-açúcar.

- Associada a esta técnica, a determinação química elementar por EDS (Energy Dispersive Spectroscopy) permitiu a ratificação do elemento identificado na imagem MEV.

- O aumento do percentual de partículas de bagaço de cana-de-açúcar, associado à matriz fibrosa de eucalipto, promoveu expressivo acréscimo no teor de sílica dos compósitos obtidos.

- Ambas as dosagens de resina praticadas não promoveram diferenças nos teores de sílica observados nos painéis.
- Pesquisas científicas devem ser intensificadas para avaliações em maior número de amostras e verificação do desgaste em equipamentos de corte proporcionado por compósitos contendo bagaço de cana-de-açúcar.

\section{AGRADECIMENTOS}

Os autores gostariam de expressar seus sinceros agradecimentos às empresas (i) Usina Açucareira São Manoel S.A, em São Manuel-SP e (ii) Duratex S.A (Área de Desenvolvimento de Processos das Unidades Agudos e Botucatu, interior de São Paulo), à Fundação de Amparo à Pesquisa do Estado de São Paulo (FAPESP) e ao Departamento de Ciências Florestais da ESALQ/USP.

\section{STATUS DA SUBMISSÃO}

Recebido: 15/09/2011

Aceito: 07/02/2012

Resumo publicado online: 22/03/2012

Artigo completo publicado: 30/06/2012

\section{AUTOR(ES) PARA CORRESPONDÊNCIA}

\section{Ugo Leandro Belini}

Departamento de Ciências Florestais, Escola Superior de Agricultura "Luiz de Queiroz" ESALQ, Universidade de São Paulo - USP, Av. Pádua Dias, 11, CP 9, CEP 13418-900, Piracicaba, SP, Brasil

e-mail: ubelini@yahoo.com.br

\section{REFERÊNCIAS}

Associação Brasileira da Indústria de Painéis de Madeira - ABIPA. Números [online]. [cited 2010 out. 13]. Available from: http://www.abipa.org.br/numeros. php.

Associação Brasileira de Normas Técnicas - ABNT. NBR 15316: Painéis de fibra de média densidade. Rio de Janeiro:ABNT; 2006.

Associação Brasileira de Produtores de Florestas Plantadas - ABRAF. Anuário estatístico da ABRAF. Brasília: ABRAF; 2010.

Athanassiadou E, Roffael E, Mantanis G. Medium density fibreboards (MDF) from recycled fibres. In: Proceedings of the Management of recovered wood: strategies towards a higher technical, economical ans 
environmental standard in Europe; 2005; Bordeaux. Bordeaux. France; 2005. p. 248-261.

Brito JO, Barrichelo LEG. Correlações entre características físicas e químicas da madeira e a produção de carvão vegetal: I. Densidade e teor de lignina da madeira de eucalipto. Instituto de Pesquisa e Estudos Florestais - IPEF 1977; 14:9-20.

Campos ACM, Hein PRG, Mendes RF, Mendes LM, Chaix G. Near infrared spectroscopy to evaluate composition of agro-based particleboards. BioResources 2009; 4(3):1058-1069.

Chen CM, Hsu LC-Y. Effects of treatment methods on relative reactivity of biomass extracts toward formaldehyde. Forest Products Journal 2002; 52(2):9299.

Centro de Tecnologia Canavieira - CTC. Bagaço e palha de cana para fins energéticos [online]. [cited 2011 ago. 11]. Available from: http://www.ctcanavieira.com.br.

Ecowood do Brasil. Produção de Painel Industrial AFB (Agricultural Fiber Board) com bagaço de cana [online]. [cited 2009 set 30]. Available from: http://www. nucleoestudo.ufla.br/necana/pdfs/aproveitamento_de_ bagaco.pdf.

Food and Agriculture Organization of the United Nations - FAO. Proceedings of the FAO advisory Committee on paper and wood products. Rotorua, New Zealand; 2000.

Forest Products Laboratory. Wood handbook: Wood as an engineering material. Madison: U.S. Department of
Agriculture, Forest Service, Forest Products Laboratory; 1999.

Mantanis G, Athanassiadou E, Nakos P, Coutinho A. A new process for recycling waste fiberboards. In Proceedings of the XXXVIII International Wood Composites Symposium; 2004; Washington. Washington State University; 2004.

Paiva JMF, Trindade WG, Frollini E. Sugar cane bagasse fiber as reinforcement in phenolic-matrix composites. In: Proceedings of the II International Symposium on Natural Polymers and Composites; 1998; Atibaia. Brasília: EMBRAPA-SPI; 1998.

Romero T. Bagaço na construção civil. Agência FAPESP; 2007. [cited 2009 nov. 21]. Available from: http://www. agencia.fapesp.br/materia/7211/noticias/bagaco-naconstrucao-civil.htm.

Rowel RM, Keany FM. Fiberboards made from acetylated bagasse fiber. Wood and Fiber Science 1991; 23(1):15-22.

União da Indústria de Cana-de-Açúcar - UNICA. Canade-açúcar processada pelas Usinas Brasileiras (Produção cana-de-açúcar no Brasil) [online]. [cited 2010 fev. 23] Available from: http://www.unica.com.br/downloads/ estatisticas/processcanabrasil.xls.

Widyorini R, Xu J, Umemura K, Kawai S. Manufacture and properties of binderless particleboard from bagasse I: effects of raw material type, storage methods, and manufacturing process. Journal of Wood Science 2005; 51:648-654. http://dx.doi.org/10.1007/s10086-005$0713-\mathrm{Z}$ 
